\title{
Aumento de productividad en una planta de trefilación de aceros para la fabricación de electrodos AWS 70S-6
}

\author{
Productivity increase of an AWS 70S-6 steel electrodes wire drawing plant \\ Alfredo Artigas $^{1 *} \quad$ Rodrigo Seco $^{1} \quad$ Alberto Monsalve $^{1} \quad$ Linton Carvajal $^{1}$ \\ Nelson Garza-Montes-de-Oca ${ }^{2}$ Rafael Colás ${ }^{2}$ \\ Recibido 21 de junio de 2016, aceptado 3 de abril de 2018 \\ Received: June 21, 2016 Accepted: April 3, 2018
}

\begin{abstract}
RESUMEN
Durante la realización de este trabajo se aunaron las fuerzas de dos empresas, una siderúrgica que es la proveedora del alambrón y la segunda es la que fabrica la soldadura en rollos continuos AWS 70S-6, fabricado básicamente en dos etapas trefilación en seco, que consta de 9 hileras aplicando reducciones desde los $6 \mathrm{~mm}$ hasta los $1.73 \mathrm{~mm}$ y en húmedo (10 hileras) desde los $1.73 \mathrm{~mm}$ hasta $0.8 \mathrm{~mm}$, sin tratamiento térmico intermedio. La motivación es de poder utilizar la capacidad instalada de la planta que es de $1000 \mathrm{Ton} / \mathrm{mes}$, previo a este trabajo la productividad es cercana a 600ton/mes. Esta baja en la productividad se atribuye principalmente a los cortes que ocurren durante el proceso de trefilado en húmedo que entorpecen la continuidad de la operación. Este trabajo tuvo como objetivo, la disminución de cortes en la etapa de trefilación en húmedo, mediante la modificación de la distribución de las tasas de reducción en trefilado en seco, minimizando los esfuerzos de trefilación y obteniendo un alambre trefilado en seco con menor límite de fluencia, esto debe repercutir en una disminución en los cortes por trefilación en el trefilado en húmedo y en consecuencia aumentar la productividad de la planta. Tras la propuesta de modificación en la distribución, se realizó una prueba industrial en la que se observó una disminución en los cortes por trefilación en húmedo y un aumento de la productividad de la planta, sumado a una disminución del consumo de hileras.
\end{abstract}

Palabras clave: Trefilación de aceros, aumento de productividad.

\section{ABSTRACT}

This work is the contribution of two industries: the first, a steel company that fabricates the wire and the second, which fabricates electrodes for welding. These electrodes, under the standard AWS 70S-6, are fabricated in a continuous process consisting of two stages: dry drawing, with nine dies applying reductions from 6 up to $1.73 \mathrm{~mm}$; the second stage is a wet drawing, with ten dies, from 1.73 up to $0.8 \mathrm{~mm}$, without heat treatment between both stages. The goal of the present work is to increase the production of the plant, from 600 ton/month up to 1,000 ton/month, by means a study of the mechanical and metallurgical variables of the process. The main reason for low productivity is the fracture of the wire during the wing process, specifically during the wet drawing stage. From this, the hypothesis of

\footnotetext{
1 Departamento de Ingeniería Metalúrgica. Facultad de Ingeniería. Universidad de Santiago de Chile. Av. L.B. O’Higgins 3363. Estación Central. Santiago, Chile.CP9160000.E-mail: alfredo.artigas@usach.cl; rodrigo.allende@usach.cl; alberto.monsalve@usach.cl; linton. carvajal@usach.cl

2 Facultad de Ingeniería Mecánica y Eléctrica. Universidad Autónoma de Nuevo León. San Nicolás de los Garza. Nuevo León, México CP 66450. E-mail: nelson.garza@gmail.com; colas.rafael@gmail.com

* Autor de correspondencia
} 
the work is that a decrease in the fracture of the wire during the wet drawing stage, it will be possible to increase the productivity of the plant, modifying the reduction rates during dry drawing, decreasing the drawing stresses and obtaining a softer dry drawn wire (lower yield stress). Lower |stresses must decrease the fracture of wires and then, increase the productivity. From the results obtained in this work, an industrial proposal was made, after which, a decrease of $45 \%$ in the fracture of wires was measured and a decrease in the consumption of dies of $50 \%$ was obtained.

Keywords: Steel wire drawing, productivity increase.

\section{INTRODUCCIÓN}

En general, una planta trefiladora costa de dos etapas un trefilado en seco y otro en húmedo, si bien es cierto que la gran problemática y cantidad de cortes y consecuencia la pérdida de productividad se produce en el trefilado en húmedo, muchos de ellos se estiman que proviene de las consecuencias del trefilado en seco. La hipótesis, por lo tanto, corresponde a que es posible disminuir la cantidad de cortes durante la etapa de trefilación en húmedo, a través de una modificación en la distribución de las tasas de reducción del trefilado en seco, provocando un producto semiterminado (trefilado en seco) con menor acritud que el actual provocando una menor frecuencia de cortes en el trefilado en húmedo y en consecuencia debería existir un aumento de la productividad de la planta. Por ello, este trabajo se enfoca en el estudio de la etapa de trefilación en seco y sus repercusiones en la frecuencia de cortes en el trefilado en húmedo.

\section{ANÁLISIS TEÓRICO}

\section{Trefilación}

Para comprender mejor este procedo, entenderemos trefilación como aquel proceso de conformado en el que mediante una carga tractiva estira una barra a través de un dado, provocando una reducción en la sección transversal [1]. La Figura 1, presenta un esquema del proceso de trefilación, en el que se reduce la sección transversal de una barra de perfil redondo desde un diámetro Di a uno Df, mediante la aplicación de una fuerza de tiro, que hace pasar la barra a través de un dado de sección cónica truncada. Por la aplicación de esta fuerza tractiva, se producen fundamentalmente dos respuestas una, del tipo compresiva y responsable de la reducción de área y otra, la fuerza de roce, en la intercara entre el dado y la barra y el dado, que se opone al movimiento de ésta.
En el proceso de trefilación influyen varios parámetros, que pueden clasificarse en dos grandes grupos, los primeros, del tipo geométrico, que son propios del material a trefilar como del dado empleado y el segundo que responde al roce, producto de la calidad superficial del producto y de la lubricación empleada.

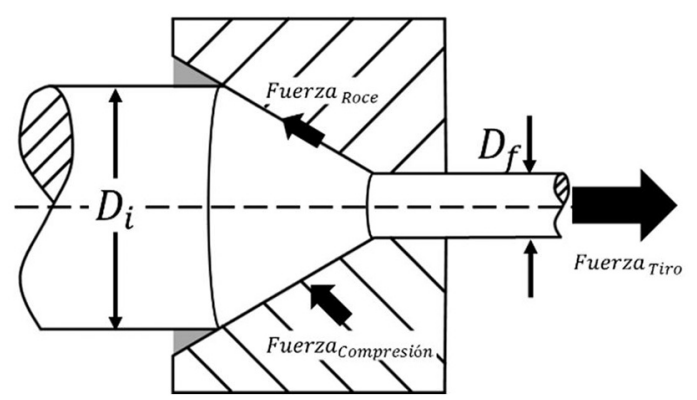

Figura 1. Esquema del proceso de trefilación.

Sea cual sea el análisis que se realice para la determinación de cómo varía la energía de trefilación $\left(\mathrm{W}_{\mathrm{T}}\right)$, en función de los parámetros de trefilación (reducción de área, ángulo del dado, límite elástico del material y coeficiente de roce) siempre parte de la premisa [2] que la energía invertida como esfuerzo, o carga, de trefilación, está compuesta por la energía interna de deformación $\left(\mathrm{W}_{\mathrm{I}}\right)$, la energía por trabajo redundante $\left(\mathrm{W}_{\mathrm{S}}\right)$ y la energía para vencer el roce que existe entre el material trefilado y el dado $\left(\mathrm{W}_{\mathrm{F}}\right)$ (ecuación 1). En la Figura 2 se puede apreciar cómo se comporta la carga relativa (esfuerzo de trefilado sobre límite de fluencia del material).

$$
\mathrm{W}_{\mathrm{T}}=\mathrm{W}_{1}+\mathrm{W}_{\mathrm{S}}+\mathrm{W}_{\mathrm{F}}
$$

De la Figura 2, se puede inferir que conforme aumenta el ángulo del dado, disminuye, en gran medida, la energía "perdida" por fricción y aumenta 


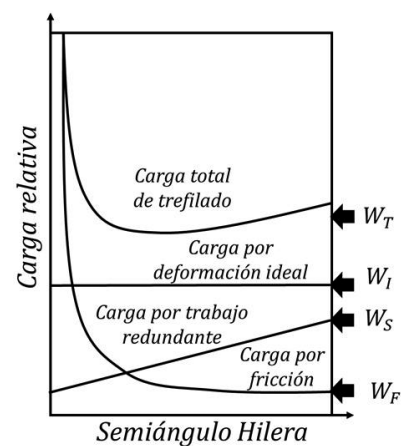

Figura 2. Carga relativa en función del semiángulo del dado.

(en menor medida) por trabajo redundante. Por ello es que se puede determinar un ángulo, en el que se minimizan estas pérdidas. Esto también se puede realizar obteniendo una expresión que relacione esfuerzo de trefilado $\left(\sigma_{\text {Trefilación }}\right)$, límite elástico $\left(\bar{\sigma}_{y}\right)$, ángulo del dado $(2 \cdot \alpha)$, coeficiente de roce $(\mu)$ y reducción de área. Un caso particular de esto es el método de Siebel (ecuación 2) [3], que surge como una solución a la Ecuación 1, considerando el método de elementos finitos,

$$
\sigma \text { Trefiliación }=\bar{\sigma}_{y} \cdot\left(\frac{2}{3} \alpha+\varepsilon+\varepsilon \cdot \frac{\mu}{\alpha}\right)
$$

Donde,

$\mathrm{A}_{\mathrm{i}}$ el área transversal de la barra sin trefilar,

y se define $\varepsilon=\ln \left(A_{i} / A_{f}\right)$.

Se ha visto en otros estudios [4] que, durante la trefilación, la deformación no es homogénea y que esta es la responsable del endurecimiento a niveles mayores que lo que dice el endurecimiento por deformación típico, estudiado a través del ajuste de Hollomon. Lo anterior se presenta de manera esquemática en la Figura 3, que presenta la evolución de las propiedades mecánicas de un alambre trefilado al sufrir sucesivamente la reducción $-r_{i}-$ de su sección transversal.

\section{PROCEDIMIENTO EXPERIMENTAL}

\section{Clasificación de cortes}

Con el propósito de aumentar productividad de la planta de trefilación, se procedió a realizar un catastro de la situación actual de ésta (trefilado en seco y húmedo), que consistió en cuantificar el

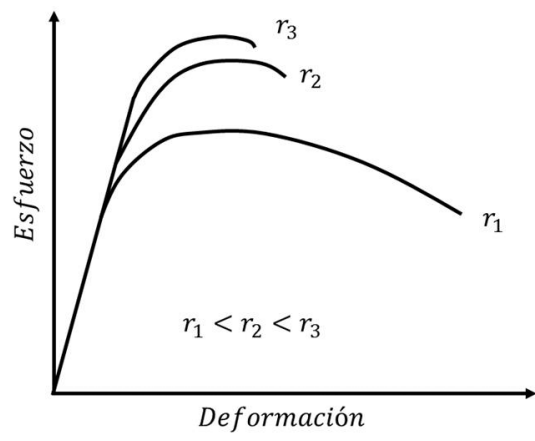

Figura 3. Evolución de la curva esfuerzo-deformación ingenieril.

desgaste de las hileras (dados de trefilación) y en clasificar los tipos de cortes en tres:

1. Falla por la unión soldada, es decir, en la unión de las bobinas o de cortes producidos.

2. Fallas de la materia prima en productos de inclusiones o, pliegues de laminación y/o incrustaciones

3. Cortes producidos por trefilación.

Una vez realizado el catastro de los cortes clasificados (de un total de 100 toneladas procesadas), los que principalmente ocurrieron en la etapa de trefilación en húmedo (últimos pases de trefilación), se procedió a realizar pruebas de trefilación en laboratorio, con el objetivo de analizar el alambre a medida que pasa por cada dado de trefilación, así, determinando la fuerza de tiro y el endurecimiento del alambre por trefilación en seco.

\section{Pruebas de laboratorio}

Los ensayos de prueba (en laboratorio) se realizaron en una máquina de tracción. Dado que el acero presenta un bajo índice de sensibilidad a la velocidad de deformación [5-7] es esperable que los resultados obtenidos en laboratorio, a una velocidad de trefilación menor a la de la planta, sean equivalentes a los que se obtendrían en esta. En la Figura 4, se presenta un esquema del montaje de trefilación realizado en laboratorio.

Luego, se propuso después de varias pruebas una nueva distribución de hileras (dados) para el trefilado en seco, con la que se pretendió lograr una disminución de los esfuerzos de trefilación y con ello obtener una baja de la resistencia del alambre (Límite 


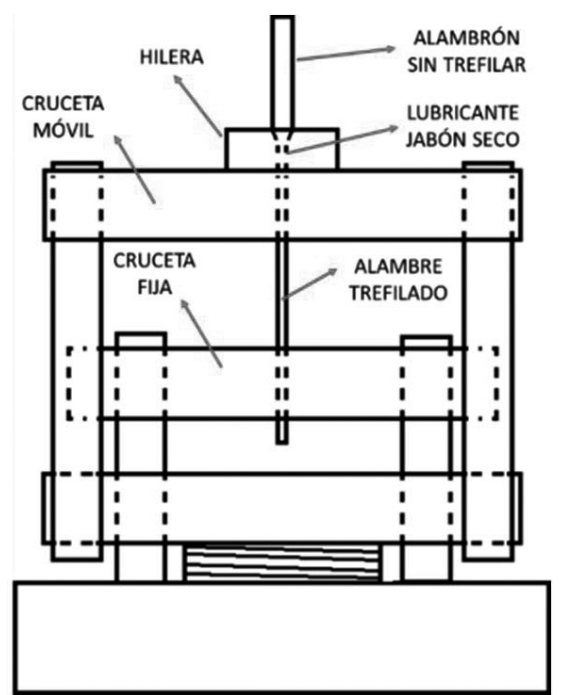

Figura 4. Esquema montaje de trefilación en laboratorio.

elástico). Al final, y con la distribución de hileras propuesta, se procedió a realizar una prueba industrial de 100 toneladas de alambrón, aproximadamente, las que poseían las mismas características (misma colada), de las que se cuantificaron los cortes, y el desgaste de las hileras, con la configuración actual y la propuesta.

\section{RESULTADOS Y DISCUSIONES}

\section{Propuesta}

Tras varias pruebas de laboratorio, se realizó una propuesta (Tabla 1), que modifica las condiciones bajo las que se realiza la trefilación en seco de la planta, en función de minimizar los esfuerzos de trefilación y endurecimiento del acero.

Se modificaron las tasas de reducción de los dados de trefilación en seco -9 en total-, en las que el diámetro de entrada $(6[\mathrm{~mm}])$ y el de salida $(1.73$ $[\mathrm{mm}])$ fuesen los mismos. Adicionalmente se cambiaron los semiángulos de las hileras.

El aumento de los semiángulos de las hileras, propuesto para las primeras pasadas, se realiza bajo la base que para ángulos pequeños (menores a $10^{\circ}$ ) existe una predominancia de la fricción y que esta promueve el desarrollo de una fuerza de tiro excesiva. Al aumentar el semiángulo de la hilera disminuye drásticamente la fricción y en respuesta a ello, la energía disponible se utiliza para aumentar la deformación por cizalle que genera un consumo energético por trabajo redundante, tal como se presentó en los antecedentes teóricos.

En la gráfica de la Figura 5, se muestran la distribución actual de la planta (línea continua), donde se destacan las reducciones en los últimos pasos, esta configuración es utilizada para evitar el cambio de todos los dados de trefilación cuando se deseaba llegar a mayores diámetros finales, es decir trabajan con 8 hileras en vez de nueve. Este procedimiento se realizó bajo la premisa de que la influencia que tiene el trefilado en seco no es relevante en la trefilación en húmedo. Además, en la misma grafica (Figura 5) se muestra una configuración distinta de reducciones para el trefilado en seco al cuantificar los esfuerzos de trefilación en laboratorio se puede observar, que en las etapas finales los esfuerzos disminuyen y

Tabla 1. Distribuciones de reducción de área y semiángulos de las hileras.

\begin{tabular}{|c|c|c|c|c|c|c|}
\hline \multirow{2}{*}{ Hilera } & \multicolumn{3}{|c|}{ Antes de la Propuesta } & \multicolumn{3}{c|}{ Después de la Propuesta } \\
\cline { 2 - 7 } & $\begin{array}{c}\text { Diámetro } \\
\text { entrada mm }\end{array}$ & $\begin{array}{c}\text { Diámetro } \\
\text { salida mm }\end{array}$ & Semiángulo $^{\text { }}$ & $\begin{array}{c}\text { Diámetro } \\
\text { entrada mm }\end{array}$ & $\begin{array}{c}\text { Diámetro } \\
\text { salida mm }\end{array}$ & Semiángulo $^{\text { }}$ \\
\hline 1 & 6,00 & 5,13 & 7 & 6,00 & 5,06 & 9 \\
\hline 2 & 5,13 & 4,43 & 7 & 5,06 & 4,30 & 9 \\
\hline 3 & 4,43 & 3,84 & 7 & 4,30 & 3,68 & 8 \\
\hline 4 & 3,84 & 3,34 & 7 & 3,68 & 3,18 & 8 \\
\hline 5 & 3,34 & 2,90 & 7 & 3,18 & 2,77 & 7 \\
\hline 6 & 2,90 & 2,50 & 7 & 2,77 & 2,43 & 7 \\
\hline 7 & 2,50 & 2,17 & 7 & 2,43 & 2,15 & 6 \\
\hline 8 & 2,17 & 1,96 & 6 & 2,15 & 1,92 & 6 \\
\hline 9 & 1,96 & 1,73 & 6 & 1,92 & 1,73 & 6 \\
\hline
\end{tabular}




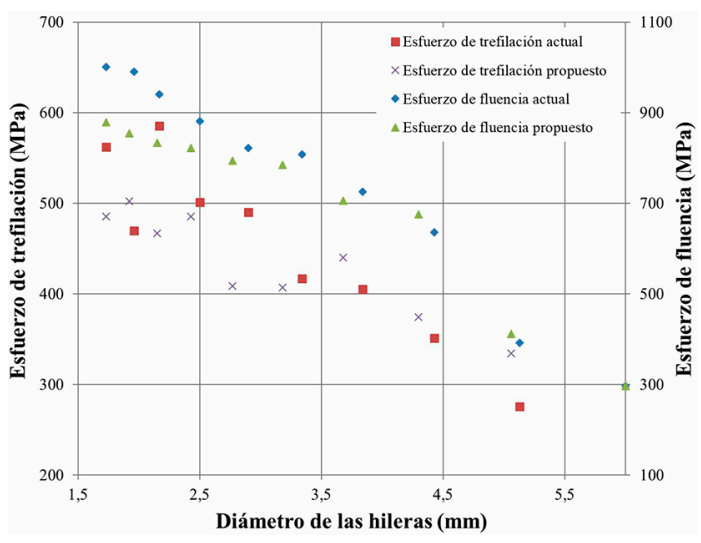

Figura 5. Esfuerzos de trefilación y reducciones.

son más constantes, con ello se espera disminuir la probabilidad de corte durante el proceso posterior del trefilado en húmedo.

En la Figura 6 se presentan límites elásticos del alambre en cada paso de trefilación, donde se observa, que con la configuración propuesta el alambre no se endurece en la misma medida que van en aumento las tasas de reducción, caso contrario a

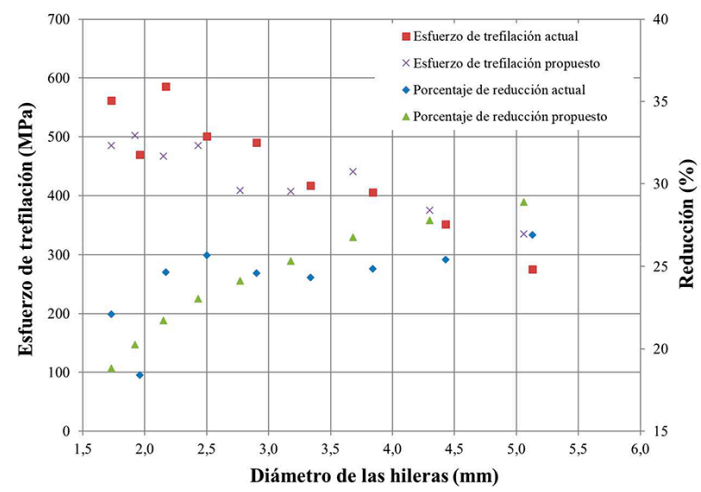

Figura 6. Esfuerzos de trefilación y límites elásticos. Antes (actual) y después de la propuesta. lo que ocurre en la configuración actual (antes de la propuesta). Lo más relevante fue que el alambre, después de la trefilación, alcanzó un límite elástico de 951 [MPa], mientras que antes de la propuesta presentaba 1059 [MPa], entregando a trefilación en húmedo un alambre más blando.

\section{Prueba Industrial}

Con el afán de corroborar los resultados obtenidos a escala de laboratorio, es que se realizaron pruebas de trefilación a nivel industrial, empleando acero de las mismas características de los que se realizó el clasificado de cortes. La comparación de los resultados obtenidos en laboratorio y los obtenidos en la prueba industrial con la configuración propuesta, se presentan en la Tabla 2. Los resultados ahí presentados corresponden al promedio obtenido tras 5 probetas ensayadas de áreas. Antes (actual) y después de la propuesta.

De la Tabla 2, se puede comentar que resultados concernientes a límites elásticos y resistencia a la tracción de las pruebas de laboratorio e industrial son concordantes el uno con el otro, corroborando lo mencionado con antelación, dado que el acero posee una baja sensibilidad a la velocidad de deformación [5-7]. Relativo a la reducción de área se aprecia que las muestras obtenidas luego de las pruebas industriales son mayores a los emanados por las pruebas de laboratorio. Lo anterior se justificaría en el aumento de temperatura que se produce a altas velocidades de trefilación [8], este aumento en la temperatura, cercana a los $100^{\circ} \mathrm{C}$ permiten un alivio de tensiones [9], lo que provocaría un aumento en la ductilidad (mayor reducción de área del material trefilado), sin disminuir de manera importante límite elástico y la resistencia máxima a la tracción.

Finalmente, se realizó una prueba industrial de trefilación en seco, con la configuración propuesta, pasando a través de las hileras 50 toneladas de

Tabla 2. Comparación de resultados de pruebas de laboratorio e industrial, antes y después de la propuesta de configuración.

\begin{tabular}{|l|c|c|c|c|c|c|}
\hline \multirow{3}{*}{ Configuración } & \multicolumn{2}{|c|}{$\begin{array}{c}\text { Límite elástico } \\
\text { MPa }\end{array}$} & $\begin{array}{c}\text { Resistencia a la tracción } \\
\text { MPa }\end{array}$ & \multicolumn{2}{c|}{$\begin{array}{c}\text { Reducción de área } \\
\%\end{array}$} \\
\cline { 2 - 7 } & Laboratorio & Industrial & Laboratorio & Industrial & Laboratorio & Industrial \\
\hline Antes de la propuesta & 1059 & 1059 & 1079 & 1079 & 42 & 44 \\
\hline Después de la propuesta & 951 & 951 & 1079 & 1079 & 49 & 57 \\
\hline
\end{tabular}


acero, de la misma colada, evaluando así su comportamiento durante el trefilado en húmedo. En la Tabla 3, se muestran los cortes por tonelada bajo la configuración antes y después de la propuesta, observándose una mejora cercana al $45 \%$, y si se considera que cada corte en trefilación en húmedo significa cerca de 25 minutos de detención de la máquina, la productividad de la planta debería haber aumentado de manera considerable.

Tabla 3. Cortes por tonelada de acero trefilado.

\begin{tabular}{|l|c|}
\hline \multicolumn{1}{|c|}{ Configuración } & $\begin{array}{c}\text { Cortes por tonelada en } \\
\text { trefilado en húmedo }\end{array}$ \\
\hline Antes de la propuesta & 0.91 \\
\hline Después de la propuesta & 0.50 \\
\hline
\end{tabular}

Con los resultados obtenidos, se tomó la decisión de cambiar la configuración de las hileras de trefilación en seco, de acuerdo con lo presentado en la propuesta. Después de dos meses de funcionamiento se pudo concluir que existió un aumento de la producción estimada cercana a las 90 toneladas mensuales, sin embargo, las variables de proceso son tan amplias, que poder evaluar de buena forma el aumento de productividad solo a esta mejora es difícil. No obstante, se evaluó el consumo de hileras en la trefilación en seco que era de 0.29 hileras por tonelada procesada y con la propuesta bajo a 0.15 hileras por tonelada procesado, es decir existe un ahorro importante en el consumo de hileras cercano al $50 \%$.

\section{CONCLUSIONES}

Se ha realizado una propuesta de modificación de la distribución de las tasas de deformación durante la trefilación en seco de un proceso industrial, basado en datos obtenidos en pruebas de laboratorio.

La propuesta emanada tras las pruebas de laboratorio fue testeada en una prueba a escala industrial, obteniendo como resultado una disminución del $50 \%$ consumo de hileras durante el proceso de trefilación en seco y una reducción del $45 \%$ del número de cortes por tonelada durante la trefilación en húmedo.

De lo anterior, es posible disminuir la cantidad de cortes por tonelada de material trefilado, durante la etapa de trefilado en húmedo, mediante la modificación de la distribución de las hileras de reducción en seco, al obtener como producto un alambre más blando. Lo anterior se tradujo en un aumento de la productividad de 90 toneladas mensuales.

\section{AGRADECIMIENTOS}

Los autores desean agradecer a los proyectos DICYT, Código 051614AA, Vicerrectoría de Investigación, Desarrollo e Innovación, y al proyecto1555LD ambos de la Universidad de Santiago.

Rodrigo Allende desea agradecer a Comisión Nacional de Investigación Científica y Tecnológica, CONICYTPFCHA/Doctorado Nacional/2017-21170167 por el financiamiento de sus estudios de postgrado.

\section{REFERENCIAS}

[1] G. Dieter. Mechanical Metalurgy, SI Metric Edition. McGraw-Hill Book Company. 3a Edición Internacional. Londres, Reino Unido. Vol. 1, pp. 635-650. 1968. ISBN: 0-07-084187-X.

[2] J. Peña. "Análisis del proceso de trefilado de tubos". Tesis para optar al grado de magíster. Pontificia Universidad Católica de Chile. Santiago, Chile. 2008.

[3] C. Luis, J. León, R. Luri. "Comparison between finite element method and analytical methods for studying wire drawing processes". Journal of Materials Processing Technology. Vol. $164 \mathrm{~N}^{\circ}$ 2, pp. 1218-1225. Date of visit: May, 2005. ISSN: 0924-0136. DOI: 10.1016/j. jmatprotec.2005.02.138.

[4] E. Rojas, D. Celentano, A. Artigas y A. Monsalve. "Simulación experimental y numérica de un proceso de trefilado húmedo de un alambre de acero al carbono" Ingeniare, Revista Chilena de Ingeniería. Vol. $16 \mathrm{~N}^{\circ}$ 1, pp. 188-194. Junio de 2008. URL: ISSN: 0718-3305. DOI: $10.4067 /$ S0718-33052008000100008.

[5] F. Gálvez, J. M. Atienza, J. Ruiz y M. Elices. "El efecto de la velocidad de deformación en la rotura de alambres de acero durante el trefilado". Anales de mecánica de la fractura. pp. 73-78. Gerona, España. 2002. 
[6] F. Gálvez, J. M. Atienza, J. Ruiz y M. Elices. "Influencia de la velocidad de deformación en el comportamiento mecánico de alambres de acero trefilados". Anales de mecánica de la fractura. pp. 185-189. Bayona, España. 2001.

[7] S. He, P. Van Houtte, A. Van Bae, F. Mei, A. Sarban, P. Boesman and F. Galvez, J. Atienza. "Strain rate effect in high-speed wire drawing process". Modelling and Simulation in Materials Science and Engineering. Vol. $10 \mathrm{~N}^{\circ}$ 3, pp. 267-276. Date of visit: March, 2002. URL: ISSN: 0965-0393. DOI: 10.1088/0965-0393/10/3/302.
[8] A. Haddi, A. Imad and G. Vega. "Analysis of temperature and speed effects on the drawing stress for improving the wire drawing process". Materials \& Design. Vol. $32 \mathrm{~N}^{\circ} 8$, pp. 4310-4315. Date of visit: April, 2011. URL: /j.matdes.2011.04.010.

[9] C. Moon and N. Kim. "Analysis of wire-drawing process with friction and thermal conditions obtained by inverse engineering". Journal of Mechanical Science and Technology. Vol. 26 N 9 , pp. 2903-42911. Date of visit: April, 2012. URL: /s12206-012-0711-1. 